Topics

\title{
Hayabusa2 - The Next Asteroid Sample Return Mission of Japan
}

\author{
By Makoto YoshikawA ${ }^{1)}$, Sei-ichiro Watanabe ${ }^{2)}$, Yuichi TsudA ${ }^{1)}$, Hitoshi KuninAKA ${ }^{1)}$ and Hayabusa2 Project Team \\ 1) JAXA, Sagamihara, Japan \\ ${ }^{2)}$ Nagoya University, Nagoya, Japan
}

(Received June 28th, 2013)

\begin{abstract}
Hayabusa2 mission has started as the follow-on mission of Hayabusa. The target asteroid is 1999 JU3, which is a C-type asteroid. The scientific purpose is to study the formation and evolution of the solar system, especially to study the organic matter and water, which existed in the early stage of the solar system formation. The engineering is also important and the spacecraft will be much more robust and reliable with some new technological challenges. The launch of Hayabusa2 is planned at the end of 2014, arriving at the asteroid in the middle of 2018, and it comes back to the earth at the end of 2020. At present, the flight model is under manufacturing.
\end{abstract}

Key Words: Planetary Exploration, Asteroid, Hayabusa

\section{Introduction}

Hayabusa is the first asteroid sample return mission in the world. Hayabusa, which was launched on May 9, 2003, arrived at the target asteroid (25143) Itokawa on September 12, 2005. We were surprised to see the strange and unexpected nature of Itokawa. After overcoming several serious problems, Hayabusa came back to the earth on June 13, 2010, and the capsule landed on the desert of Australia successfully. Although Hayabusa had a lot of troubles and difficulties, it was successful to bring back the surface materials of Itokawa. The initial sample analysis has already been done, and we revealed a little about the birth of Itokawa. At present, the samples are being analyzed by a lot of scientists in the world.

After Hayabusa, we have started next asteroid sample return mission, Hayabusa ${ }^{1)}$. Hayabusa2 is similar to Hayabusa, but the target asteroid is C-type, which is different from S-type Itokawa. From the science point of view, C-type asteroids are quite important for the next target of asteroid sample return missions. As for the engineering, many parts in the spacecraft will be modified so that Hayabusa2 will not have the same troubles occurred in Hayabusa. After several year considerations, Hayabusa2 project shifted to Phase-B in 2011 and at present (April 2013) the flight model is under manufacturing (Fig.1). The launch will be at the end of 2014. The details about the mission will be shown in the following sections.

Here, we summarize the importance of explorations of small solar system bodies. There are several purposes for exploration of the small solar system bodies. The small solar system bodies, such as asteroids and comets, are not outstanding except that comet sometimes shows long tail near the sun. However, the total number of these small bodies is quite large, and at present about 600,000 asteroids have been found. These objects are supposed to have information at the time of the birth of the solar system. Therefore, the study about the formation and evolution of the solar system is the one of the major purposes of the exploration to the small solar system bodies.

Another important thing is spaceguard. Asteroids or comets may collide to the earth. If such collisions occur, we will have large disaster. So we must prevent such collision. In order to do this, at first we must discover the objects that will come close to the earth or that have possibilities to collide to the earth. If we find such objects, then we must try to do something to avoid the collision. We must know the characteristics of colliding objects to avoid their collision. These are the activities of the spaceguard, and asteroid exploration is quite important to know the nature of such colliding objects.

Small bodies may have such materials like metal or water. These materials are necessary when mankind goes into the interplanetary space. Of course, these materials cannot be used

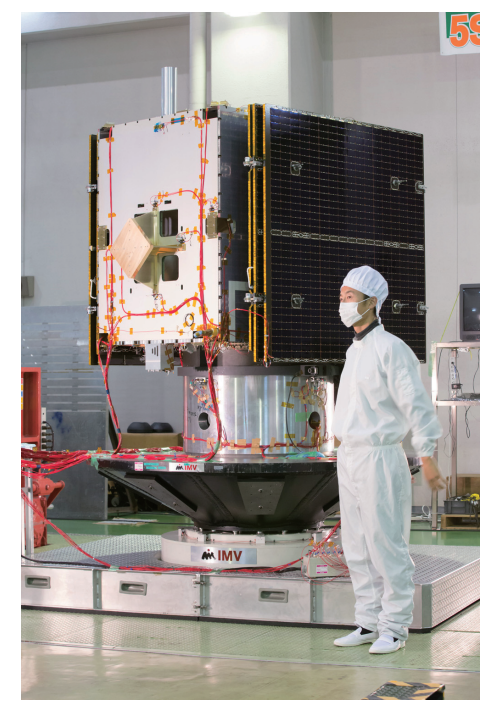

Fig. 1. Hayabusa2 flight model under manufacturing. (26 December 2012) 
right now, because it costs a lot to get them form asteroids or comets to the earth. However, in the future, it may be possible that such materials are utilized. In addition to these, the asteroids that approach to the earth have another important aspect. Such asteroids can be the targets of manned mission. Human beings have already been to the moon, and it is said that the next target is Mars. However, Mars is too far form the earth to send manned spacecraft in near future. Asteroids approaching to the earth will be the good targets before manned mission to Mars.

The small solar system bodies have such various kinds of importance. This is the reason why we are carrying out the space missions to them.

\section{Hayabusa2 Mission}

In Japan, we have several categories of space missions to the solar system bodies, such as lunar mission like "Kaguya (SELENE)", planetary missions like "Nozomi (PLANET-B)" and "Akatsuki (PLANET-C)", and missions to small solar system bodies like "Sakigake (MS-T5)", "Suisei (PLANET-A)", and "Hayabusa (MUSES-C)". Hayabusa2 is the next mission to the small solar system bodies.

\subsection{History}

The Hayabusa2 mission was proposed in 2006 at first. In the year before this, 2005, Hayabusa tried to get the surface material from Asteroid Itokawa, but it could not do this as planned. Moreover Hayabusa had very serious problems, and we were not sure whether it could come back to the earth or not. Therefore, we proposed Hayabusa 2 to try again. Asteroid 1999 JU3 was selected as the target object for Hayabusa2, because it is C-type asteroid. Since Asteroid Itokawa is S-type, we thought that we would have much more new scientific results if we explore a different kind of asteroid.

In this first proposal, the spacecraft was almost same as that of Hayabusa, because we wanted to start it as soon as possible. Of course, we would modify the parts where trouble occurred in Hayabusa, but there were no major changes. The launch windows to go to 1999 JU3 were in 2010 and 2011. However, since we could not start Hayabusa 2 mission immediately, we missed these launch opportunities. The next launch windows are in 2014 and 2015. Therefore, we have changed our original plan and we are now planning to launch Hayabusa2 in 2014. Since the launch was delayed, we changed our plan a little. We put a new instruments, such as Ka-band antenna and what we call "impactor." These new instruments are explained in the later section.

In May 2011, the status of Hayabusa2 project shifted to Phase-B, and we have stated to develop the spacecraft of Hayabusa2 for the launch in 2014. The critical design review
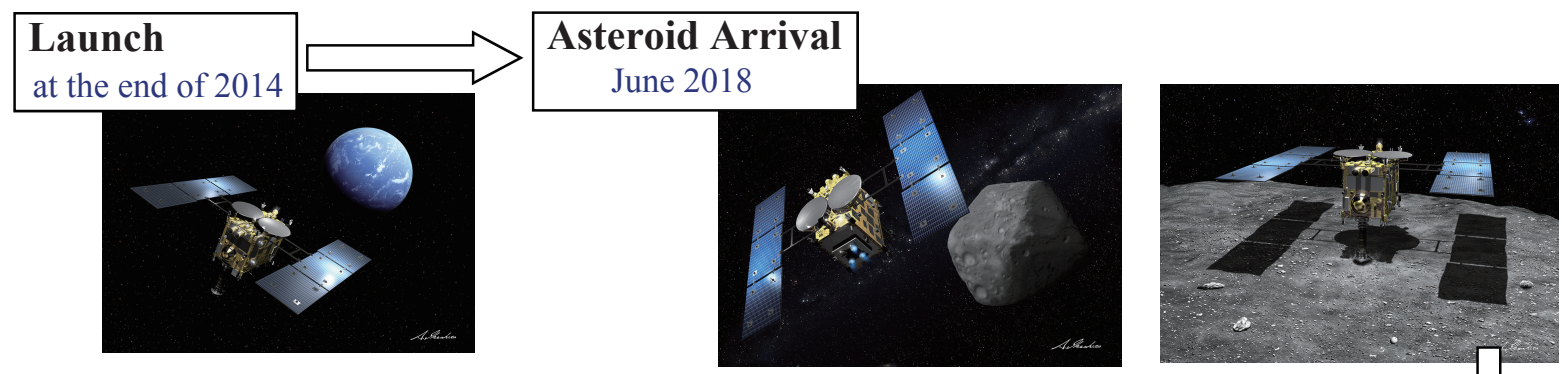

Hayabusa2 observes the asteroid by the optical camera, the near infrared spectrometer, Laser altimeter, and etc. Then it approaches near the surface of the asteroid, releases the rovers and the lander, and tries to get the surface material.

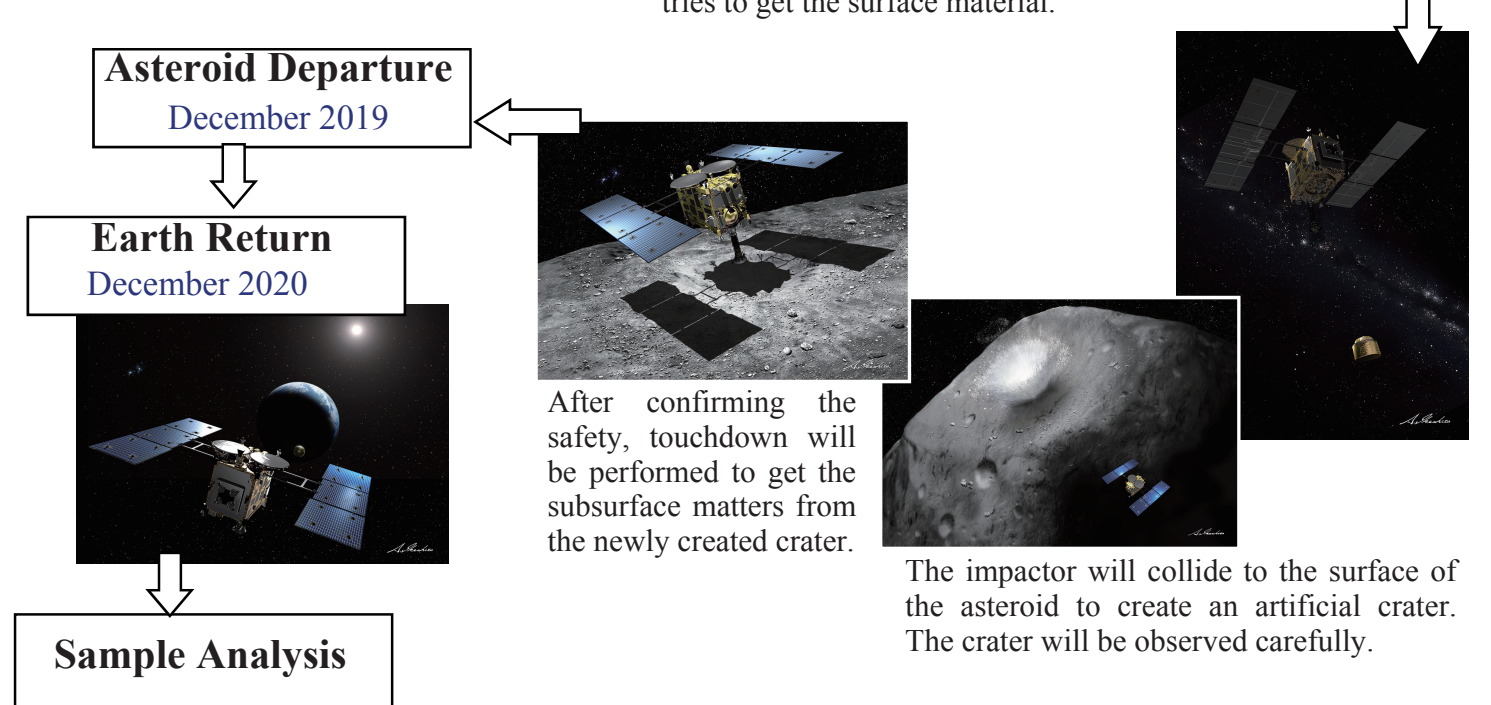

Fig. 2. The current scenario of Hayabusa 2 mission. 
(CDR) was finished in March 2012, and we started manufacturing the flight model. The first interface test is on going now (April 2013).

\subsection{Mission scenario}

The launch window that we are aiming at now is at the end of 2014. We use H-IIA rocket to launch Hayabusa2. The launching site is in Tanegashima Island in Japan. There are other launch windows in 2015, which are backup windows. After the launch, the spacecraft comes back to the earth in December 2015 to execute the earth swing-by, and departs from the orbit near the orbit of the earth.

In the cruising phase, Hayabusa 2 uses the ion engine, which is the modified one that was used for Hayabusa. Hayabusa2 will arrive at 1999 JU3 in June 2018. It will stay near the asteroid for about one and half years. This period is much longer than the case of Hayabusa. Hayabusa had only three months to explore Itokawa and this was too short to do the many missions.

At the asteroid, Hayabusa2 observes the asteroid in detail at first. Next it releases small rovers and a lander. Then Hayabusa2 performs touchdown to collect the surface material. The method of sample correction is almost same as that of Hayabusa. Finally, we use the impactor to make a small crater and touchdown again to the crater to collect the material revealed from the subsurface.

Hayabusa2 will leave the asteroid at the end of 2019, and come back to the earth at the end of 2020 . The capsule will be released and it will land on the desert in Australia. The spacecraft will flyby near the earth and go somewhere. The capsule will be brought back to Japan and it will be opened in the curation facility in JAXA. We hope that we will find the sample of 1999 JU3 in it. If we find some samples then the initial analysis will be done by the science team of Hayabusa2 first. And later, the samples will be distributed to researchers in the world.

The whole scenario is summarized in Fig.2.

\subsection{Spacecraft and payloads}

As we already mentioned, the spacecraft of Hayabusa2 is basically similar to that of Hayabusa. However, we have the experience of Hayabusa, so we have improved many components of spacecraft so that the mission will be more reliable and robust. For example, Hayabusa had three reaction wheels, and two of them were broken before the touchdown. Therefore, we put four reaction wheels on Hayabusa2, so Hayabusa2 is more redundant in the attitude control. As for the ion engine, it is modified so that it is more durable and its thrust level becomes a little higher. The chemical thruster system is also modified. The layout the pipes of the fuel and the oxidizer is changed so that we can avoid the troubles that occurred in Hayabusa and Akatsuki (PLANET-C), the Venus missions of Japan.

One of the new sub-systems for Hayabusa2 is Ka-band communication. Since it is better to have high-rate downlink, we have Ka-band $(32 \mathrm{GHz})$ in addition to X-band $(8 \mathrm{GHz})$. If we use Ka-band, we can send data about four times faster than the X-band can do. Ka-band will be used when we download the observed data of 1999 JU3.

Another new thing is the small carry-on impactor (SCI). This impactor is a small box that contains explosive. After released from the spacecraft about a few hundred meters above the surface of the asteroid, the impactor will explode and a lump of copper will be accelerated at the speed of 2 $\mathrm{km} / \mathrm{s}$ or so. The mass of the copper is about $2 \mathrm{~kg}$, so we think that a crater about a few meters in diameter will be created on the surface. The purpose of the impactor is to remove the material covered the surface of the asteroid and to expose the subsurface material. Then we will try to get the subsurface materials. The surface material may have the effects of the space weathering, but the subsurface material may not. If we can get both materials, we will know much better about the original materials of the solar system. The reason why we use copper projectile is to make it easy to distinguish original matters of the asteroid from the matters of spacecraft.

As for the guidance and navigation in the proximity phase, we consider more precise method than Hayabusa using the experience of Hayabusa. For example, Hayabusa2 has five target makers while Hayabusa had only three. Two of them will be used at the first and the second touchdown. This is the

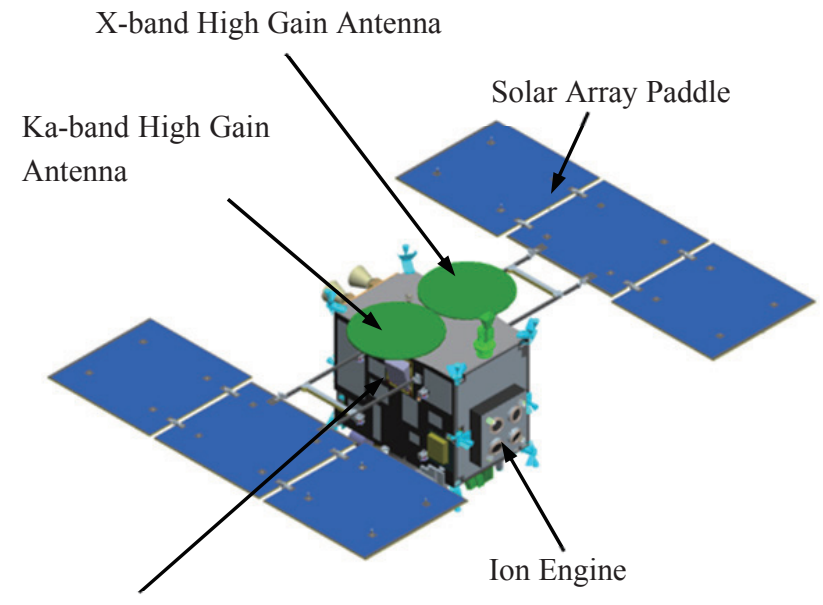

Small Lander : MASCOT

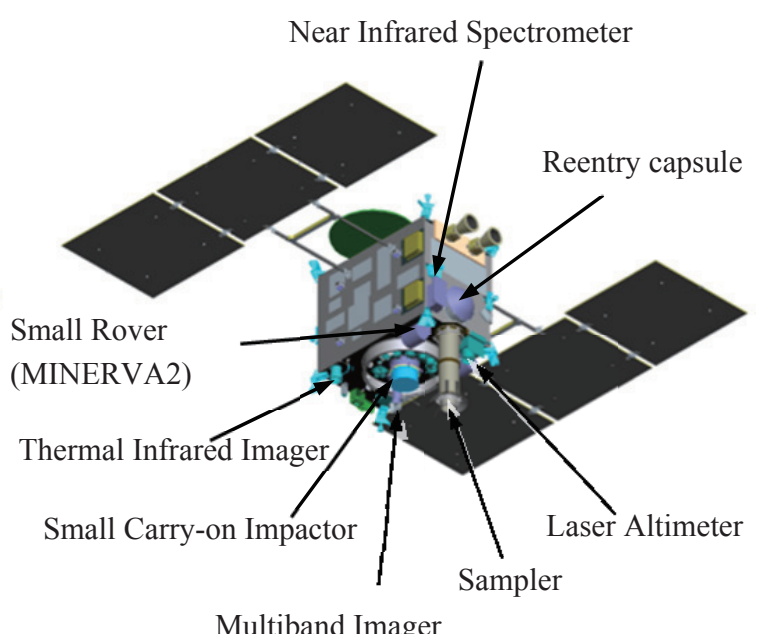

Multiband Imager

Fig. 3. Hayabusa2 spacecraft. 
Table 1. Payloads of Hayabusa2.

\begin{tabular}{|l|l|}
\hline Payloads & Specifications \\
\hline $\begin{array}{l}\text { Multiband Imager } \\
\text { (ONC-T) }\end{array}$ & $\begin{array}{l}\text { Wavelength: } 0.4-1.0 \mu \mathrm{m}, \\
\text { FOV: 5.7 deg x 5.7 deg, Pixel } \\
\text { Number: } 1024 \times 1024 \mathrm{px}, \text { filter } \\
\text { (ul, b, v, w, } \mathrm{x}, \mathrm{p}, \text { Wide) }\end{array}$ \\
\hline $\begin{array}{l}\text { Near IR Spectrometer } \\
\text { (NIRS3) }\end{array}$ & $\begin{array}{l}\text { Wavelength: 1.8 }-3.2 \mu \mathrm{m}, \\
\text { FOV: 0.1 deg x 0.1 deg }\end{array}$ \\
\hline $\begin{array}{l}\text { Thermal IR Imager } \\
\text { (TIR) }\end{array}$ & $\begin{array}{l}\text { Wavelength: } 8-12 \mu \mathrm{m}, \mathrm{FOV}: \\
12 \text { deg } \mathrm{x} 16 \text { deg, Pixel Number: } \\
320 \mathrm{x} 240 \mathrm{px}\end{array}$ \\
\hline $\begin{array}{l}\text { Laser Altimeter } \\
\text { (LIDAR) }\end{array}$ & $\begin{array}{l}\text { Measurement Range: } 30 \mathrm{~m}-25 \\
\text { km }\end{array}$ \\
\hline Sampler & $\begin{array}{l}\text { Minor modifications from } \\
\text { Hayabusa }\end{array}$ \\
\hline $\begin{array}{l}\text { Small Carry-on } \\
\text { Impactor }\end{array}$ & $\begin{array}{l}\text { Small, deployed system to } \\
\text { make an artificial crater on the } \\
\text { surface }\end{array}$ \\
\hline $\begin{array}{l}\text { Separation Camera } \\
\text { (DCAM) }\end{array}$ & $\begin{array}{l}\text { Small deployable camera to } \\
\text { observe the SCI operation }\end{array}$ \\
\hline $\begin{array}{l}\text { Small Rovers } \\
\text { (MINERVA2) }\end{array}$ & $\begin{array}{l}\text { Almost same as MINERVA of } \\
\text { Hayabusa }\end{array}$ \\
\hline $\begin{array}{l}\text { Small Lander } \\
\text { (MASCOT) }\end{array}$ & \begin{tabular}{l} 
Provided by DLR \\
\hline
\end{tabular} \\
\hline
\end{tabular}

same as the touchdown of Hayabusa. The remaining three will be used at the touchdown to the small crater that impactor makes. Since the crater is very small (a few meters in diameter), the spacecraft will try to approach step by step by using three target makers.

The Hayabusa2 spacecraft is shown in Fig.3. The size is almost the same as Hayabusa, and the wet mass is $600 \mathrm{~kg}$ or less, which is a little heavier than Hayabusa $(520 \mathrm{~kg})$.

The mission payloads of Hayabusa2 are summarized in Table 1. As for the science, we have four instruments; Multiband Imager (ONC-T), Near IR Spectrometer (NIRS3), Thermal IR Imager (TIR), and Laser Altimeter (LIDAR). ONC-T and LIDAR are almost same as those of Hayabusa, although the filter of ONC-T is changed slightly. NIRS3 is the same as NIRS of Hayabusa, but the wavelength is different. Since we want to study the absorption by water, NIRS3 must observe $3 \mu \mathrm{m}$ wavelength. TIR is new for Hayabusa2, but we have already developed it for Akatsuki. We have chosen TIR instead of X-ray fluorescence Spectrometer (XRS), which Hayabusa had. TIR is more effective to study C-type asteroid, because we can get the thermal data, which is very important to study the thermal effects on the materials of C-type asteroid.

Other mission payloads are the sampler and the two small rovers (MINERVA2), which were the payloads of Hayabusa. They will be a little changed from those of Hayabusa, but the basic functions are similar. The completely new payloads are SCI, which is already mentioned, and Separation Camera (DCAM). DCAM will be released just after the SCI separation, and it will try to get the images at the moment of the impactor collision. Another new payload is a small lander called MASCOT, which is mentioned in the next sub-section.

\subsection{Inernational collaborations}

Hayabusa 2 mission has three international collaborations. One is the collaboration with DLR (Deutsches Zentrum für Luft- und Raumfahrt) and CNES (Le Centre national d'études spatiales). DLR and CNES provide a small lander called MASCOT (Mobile Asteroid Surface Scout). MASCOT will have four science payloads to investigate the surface properties of $1999 \mathrm{JU} 3$. These four payloads are Wide angle visual camera (CAM), MicrOmega, which is a near-infrared imaging spectrometer/microscope for mineralogy and composition, Radiometer (MARA) for the measurement of the surface thermal properties, and Magnetometer (MAG) for characterization of the magnetization of the asteroid.

The second collaboration is with NASA (The National Aeronautics and Space Administration). The collaboration with NASA was done for Hayabusa, so similar collaboration is under consideration for Hayabusa2.

The third collaboration is with Australia for the capsule reentry. This collaboration was also done for Hayabusa mission.

\section{Target of Hayabusa2}

As mentioned in the previous sections, the target of Hayabusa2 is Asteroid (162173) 1999 JU3, which is C-type asteroid. The observation campaign for this asteroid was done in 2007-2008 and 2011-2012. We know the physical parameters of 1999 JU3 as shown in Table 2. The size is about $900 \mathrm{~m}$ in diameter, so it is larger than Itokawa but still it is very small object. The shape estimated up to now is rather spherical (Fig. 4), not so elongated like Itokawa. The spin period is about 7.6 hours and this is rather shorter than Itokawa, the spin period of which is about 12 hours. However, for the purpose of the sample collecting, this spin rate is no problem. The problem is that we have not determined the orientation of the spin axis yet. Since the shape of 1999 JU3 is spherical, it is very difficult to determine the spin axis. The albedo of 1999 JU3 is small, because it is C-type asteroid.

Fig. 5 shows the orbit of 1999 JU3. The orbit is similar to that of Itokawa, and it is orbiting from just inside the orbit of the earth to just outside the orbit of Mars. The inclination of the orbit is small like Itokawa. Such orbit is suitable for a small spacecraft like Hayabusa2 to reach and go back to the earth.

Table 2. Physical parameters of Asteroid 1999 JU3.

\begin{tabular}{ll}
\hline Parameter & Value \\
\hline Spin period & $7.625 \pm 0.003$ hours \\
Spin axis & difficult to estimate \\
Ratio of axis & almost spherical $^{2)}$ \\
Size & $0.87 \pm 0.03 \mathrm{~km}^{3)}$ \\
Albedo & $0.070 \pm 0.0006^{3)}$ \\
Magnitude & $\mathrm{H}=18.69 \pm 0.07, \mathrm{G}=-0.09 \pm 0.03^{2)}$ \\
Type & $\mathrm{Cg}$
\end{tabular}

These data were reported by [1] and [2]. 


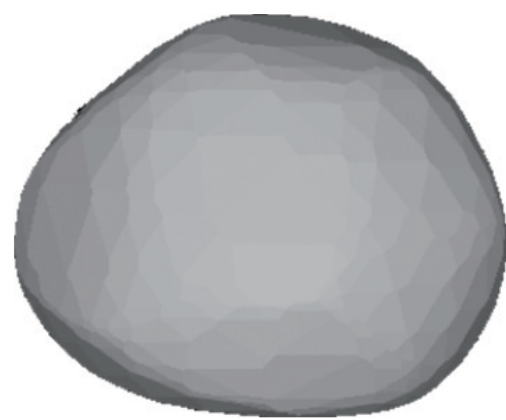

Fig. 4. Estimated shape of 1999 JU3 based on Müller model.

\section{Conclusion}

In Japan, we do not have so many planetary missions, but most of our missions are very ambitious and also very advanced. Hayabusa is the most ambitious mission among them, and we have tried many new technologies that have not been tried yet in the world. Hayabusa gave us a lot of very precious experiences. Although there were many unexpected discoveries and troubles during the mission of Hayabusa, we could manage the mission up to the final stage. Based on such experiences, we are planning the next mission, Hayabusa2. Hayabusa2 will do the similar mission as Hayabusa, but its technology will be (and should be) much more mature than that of Hayabusa. This is the most important purpose of Hayabusa2 from the point of engineering.

Another important point is the science. Although the main purpose of Hayabusa mission is not science but technology, the results of the scientific study of Itokawa attracted considerable attention from a lot of researchers, because it was the first time that we investigate such a small object of the solar system. We can say that we saw the building block of planets. The first science results were reported in the special issue of the journal of Science in $2006^{4)}$ and the first sample analysis results were also reported in the special issue of Science in 2011). As for Hayabusa2, science is quite important as well as engineering. Therefore, we chose a C-type asteroid 1999 JU3 as the mission target of Hayabusa2. We expect that we can study organic matters and waters at the beginning of the solar system. Asteroid 1999 JU3 is also small object and it is one of the near earth objects. Therefore the

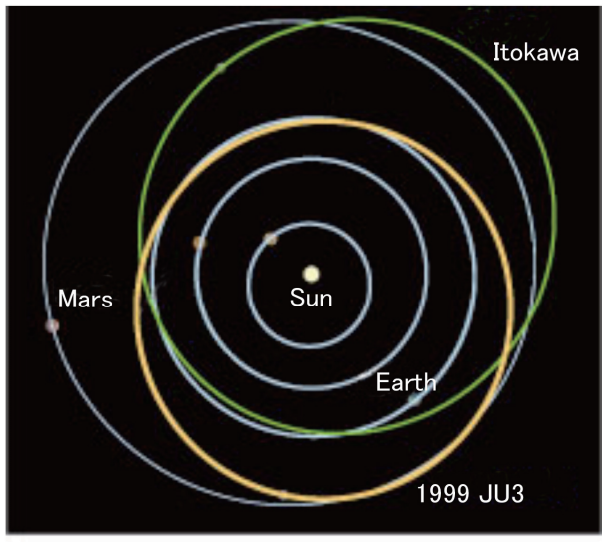

Fig. 5. The orbit of 1999 JU3.

study about small near earth objects will be much advanced by Hayabusa2, which will surely contribute to the spaceguard activity.

The small solar system bodies still have a lot of mysteries and potentials for the human in future. We are sure that Hayabusa2 will contribute a lot. After Hayabusa2, we want to plan the next mission, which may totally different from Hayabusa and Hayabusa2 so that we can study the origin and evolution of the solar system much further. Hayabusa was the starting point to the new exploration of the solar system, and Hayabusa2 is the next step, and we hope post-Hayabusa2 will follow. We are on the way to the full understanding of our solar system.

\section{References}

1) Yoshikawa, M. : Hayabusa2 Project - Its New Challenge toward Asteroid Exploration, Aeronautical and Space Sciences Japan, 60 (2012), pp.455-460 in Japanese.

2) Kim et al. : DPS Mtg. 44 (2012) \#110.03

3) Müller, T. G., Durech, J., Hasegawa, S., Abe, M., Kawakami, K., Kasuga, T., Kinoshita, D., Kuroda, D., Urakawa, S., Okumura, S., Sarugaku, Y., Miyasaka, S., Takagi, Y., Weissman, P. R., Choi, Y.-J., Larson, S., Yanagisawa, K. and Nagayama, S. : Thermo-physical properties of 162173 (1999 JU3), a potential flyby and rendezvous target for interplanetary missions, Astronomy \& Astrophysics Manuscript no.15599, November 24 (2010).

4) Hayabusa at Asteroid Itokawa, Science, 312 (2006), pp.1327-1353.

5) HAYABUSA Dust from Itokawa, Science, 333 (2011), pp.1113-1131. 